Anal ysi s of Mul ti pl e havegui de Di scont i nui t i es Usi ng Propagat i on Oper at or Met hod and Beam Pr opagat i on Net hod

\begin{tabular}{|l|l|}
\hline 著者 & MORI MOTO Kei ta, TSUJI Yasuhi de \\
\hline $\begin{array}{l}\text { j our nal or } \\
\text { publ i cat i on t i t l e }\end{array}$ & I EEE Jour nal of Quant um El ect r oni cs \\
\hline vol une & 55 \\
\hline number & 4 \\
\hline page r ange & 6100108 \\
\hline year & 2019-08 \\
\hline URL & ht t p: //hdl . handl e. net /10258/00010012 \\
\hline
\end{tabular}




\title{
Analysis of Multiple Waveguide Discontinuities Using Propagation Operator Method and Beam Propagation Method
}

\author{
Keita Morimoto and Yasuhide Tsuji, Member, IEEE, Member, OSA
}

\begin{abstract}
We apply an analysis scheme combining propagation operator method (POM) and beam propagation method (BPM) to waveguide discontinuity problems. In this approach, finite element method (FEM) with higher adaptability to waveguide geometry is utilized for discretizing waveguide cross section. While BPM based on FEM (FE-BPM) can efficiently analyze waveguides with long propagation distance, it encounters serious degradation of accuracy when waveguides with discontinuous structure has to be analyzed. The presented method overcomes this defect by applying POM which can analyze discontinuous facets and sufficiently cover various mode coupling.
\end{abstract}

Index Terms-Waveguide discontinuities, propagation operator method, beam propagation method, finite element method, Denman-Beavers iterative scheme.

\section{INTRODUCTION}

Recently, a lot of high-performance optical waveguide devices have been developed to realize high speed and large capacity optical communication systems. Among them, since splicing loss caused by the butt-coupling between different kind of optical waveguides greatly affects the deterioration of the device performance, it is necessary to evaluate the characteristics of the reflection and transmission at the connection facets properly. On the other hand, the discontinuous structures cause a problem even in the design of optical devices. Topology optimization techniques, for example, can design high performance optical devices with unpredictable waveguide structures automatically and have been actively developed in recent years [1]-[3]. However, they require enormous computational cost in particular for designing longlength devices because it is necessary to repeat the iterative calculation of analyzing the propagation characteristics until convergence condition is satisfied or the maximum iteration number is reached. Efficient topology optimization methods using beam propagation method (BPM) for propagation analysis has also been proposed [4], [5]. However there is a problem that ordinary BPM can not handle reflected waves caused by discontinuous structures. In order to solve these problems, efficient analysis of discontinuous structure is indispensable.

Direct numerical approach such as finite element method (FEM) [6], [7] and finite difference time domain (FDTD) method [8] have been widely used for calculation of various

K. Morimoto and Y. Tsuji are with the Division of Information and Electronic Engineering, Muroran Institute of Technology, Muroran, 050-8585 Japan (e-mail: 19096015@mmm.muroran-it.ac.jp; y-tsuji@mmm.muroranit.ac.jp). optical waveguides, including discontinuity problems. However, these techniques are not desired for efficient analysis because a large number of discrete meshes are required. On the other hand, bidirectional eigenmode propagation (BEP) method [9]-[11] can evaluate reflection and transmission characteristics by discretizing only the waveguide cross-section. However it is not so readily to analyze the problems with complicated structures because a lot of radiation and evanescent modes must be taken into account and large computational cost is required for mode expansion. Therefore, it has been known that propagation operator method (POM) [12]-[15] is one of the best effective approaches for evaluating the propagation characteristics at optical waveguide discontinuity. POM can calculate both reflected and transmitted field including radiation mode at the discontinuous facets just analyzing the waveguide cross-section boundary without mode expansion. In the past studies of POM, finite difference method (FDM) is usually employed to discretize the waveguide cross-section, and these recent works in [14], [15] show that they are competent to analyze including evanescent modes in a full-vectorial analysis. More recently, we investigated that POM based on FEM which is employed as alternative discretization scheme to make the discrete meshes more flexible, and demonstrated its highly accurate analysis in two- and three-dimensional waveguide problems [16], [17]. In POM analysis, it is assumed that all the structures in the propagation direction excluding the discontinuous boundary are uniform. This fact suggests that analysis of multiple discontinuities is difficult in ordinary POM.

A simple approach for analyzing the multiple discontinuous structures using POM can deal with only a structure uniform in the propagation direction between discontinuous boundaries. This method is categorized into a bidirectional-BPM (Bi-BPM) in a broad sense. As Bi-BPMs, several iterative and non-iterative techniques have been proposed in the past studies [18]-[24]. In terms of the iterative approaches for the multiple discontinuities problems, BPM is applied alternately to forward and backward waves between discontinuous facets, and the electromagnetic fields at the end of the facets are updated by appropriately adding up the contributions [24]. This process has to be repeated until a certain convergence criterion is satisfied, in addition, it is usually difficult to deal with interference of light and significant accuracy deterioration may occur in the analysis of strongly guiding waveguides. On the other hand, in non-iterative approaches, by creating transfer matrices, it is possible to efficiently analyze 
multiple discontinuities problems including interference of light between the discontinuous structures without iterative beam propagation analysis. In this case, in order to obtain the transfer matrices, the exponential matrix operator has to be approximated by some polynomial form of the matrix. Although, in the BEP, the transfer matrices can be constructed without using such an approximation, all or a sufficiently large number of the eigenmodes at each waveguide cross-section has to be evaluated. Moreover, methods based on mode expansion such as BEP, unlike field-based techniques such as POM and $\mathrm{BPM}$, require reconstruction of the transfer matrix, which is usually expensive, if the structure continuously varies between discontinuities.

Although the BPM based on slowly varying envelope approximation [25]-[28] can not consider the reflected wave, it is very useful for propagation analysis where the structure continuously varies in the propagation direction. The combined method with BEP and BPM has been also proposed, however, the BPM is independently applied to the incident, reflected and transmitted wave before and after BPM calculation [29]. Noniterative bidirectional BPM based on FEM, which does not require mode expansion, has been reported [19], however, in this approach, although fundamental and radiated backward waves are considered, the radiated transmitted fields are ignored and its applicability is limited to simple grating structures.

In this paper, an analysis scheme combining POM and BPM to multiple waveguide discontinuity problems is demonstrated in two-dimensional problems. In our method, discontinuous facets are analyzed by POM and the interval region where structure continuously varies are efficiently calculated by BPM. Once the discontinuous structures are analyzed by POM, it is possible to efficiently calculate the propagating field in several waveguide devices with different structure between the discontinuities because reconstruction of the operator matrix is not required. In addition, FEM with higher adaptability to waveguide geometry is utilized for discretizing waveguide cross-section. We investigate the validity of the proposed method by some numerical analysis examples.

\section{Combined Method of POM AND BPM}

\section{A. Basic Equation}

We consider a two-dimensional (2-D) optical waveguide connected to a discontinuity cross-section which is shown in Fig. 1, where $x$ and $y$ are the transverse directions, and $z$ is the propagation direction. In order to suppress spurious reflections of radiated waves toward $y$ direction from the artificial boundaries, perfect matched layer (PML) [30] is placed at the ends of the computational domain. Assuming that there is no variation in the $x$ direction $(\partial / \partial x)$, we obtain the following basic equation:

$$
\frac{\partial}{\partial y}\left(\frac{p}{s_{y}} \frac{\partial \Phi}{\partial y}\right)+\frac{\partial}{\partial z}\left(s_{y} p \frac{\partial \Phi}{\partial z}\right)+k_{0}^{2} s_{y} q \Phi=0
$$

where $k_{0}$ is the free space wavenumber, and $\Phi, p$ and $q$ are given by

$$
\begin{array}{llll}
\Phi=E_{x}, p=1, & q=n^{2} & \text { for } & \text { TE modes } \\
\Phi=H_{x}, p=1 / n^{2}, q=1 & \text { for } & \text { TM modes }
\end{array}
$$

where $E_{x}$ and $H_{x}$ are $x$ component of electric and magnetic fields, respectively, and $n$ is the refractive index. $s_{y}$ is the PML parameter and is given by

$$
s_{y}=1-j\left(\frac{\rho}{d}\right)^{2} \tan \delta
$$

where $d$ is the thickness of PML, $\rho$ is the distance from the beginning of PML (PML surface), and $\delta$ is the loss angle at the end of PML $(\rho=d)$.

\section{B. POM Analysis}

Considering light wave propagates along the $z$ direction with propagation constant $\beta$, and substituting a solution of the form

$$
\Phi(y, z)=\phi(y) \exp (-j \beta z)
$$

into (1), we obtain the following equation:

$$
\frac{\partial}{\partial y}\left(\frac{p}{s_{y}} \frac{\partial \phi}{\partial y}\right)+\left(k_{0}^{2} s_{y} q-s_{y} p \beta^{2}\right) \phi=0
$$

Then, applying FEM based on Galerkin method to the waveguide cross-section, we obtain the following matrix equation:

$$
\left([K]-\beta^{2}[M]\right)\{\phi\}=\{0\}
$$

where $\{0\}$ is a null vector, and $\{\phi\}$ is the transverse field components, the finite element matrices $[K]$ and $[M]$, are given by

$$
\begin{aligned}
{[K] } & =\sum_{e} \int_{e}\left[k_{0}^{2} s_{y} q\{N\}\{N\}^{T}\right. \\
& \left.-\frac{p}{s_{y}} \frac{d\{N\}}{d y} \frac{d\{N\}^{T}}{d y}\right] d y \\
{[M] } & =\sum_{e} \int_{e} s_{y} p\{N\}\{N\}^{T} d y
\end{aligned}
$$

where $\{N\}$ is the shape function vector and $\sum_{e}$ extends over all the different elements.

When the electromagnetic field $\phi$ is regarded as an arbitrary field which is not certain eigenmode, $z$-dependence of light propagation cannot be expressed as $\exp (-j \beta z)$. Thus, $\beta$ in (6) is replaced by $j d / d z$ considering $z$ direction dependence, and we obtain the following matrix form second-order ordinary differential equation:

$$
\frac{d^{2}\{\Phi\}}{d z^{2}}+[Q]^{2}\{\Phi\}=0
$$

where $[Q]$ is the propagation operator matrix which is defined by $\sqrt{[M]^{-1}[K]}$, and this square root matrix is efficiently calculated by Denman-Beavers iterative method [15] in which not combersome mode expansion is not required.

As a solution of this differential equation, the electromagnetic field can be formally expressed as

$$
\{\Phi\}=\left\{\phi^{(+)}\right\} e^{-j[Q] z}+\left\{\phi^{(-)}\right\} e^{j[Q] z}
$$

where $\left\{\phi^{(+)}\right\}$and $\left\{\phi^{(-)}\right\}$represent column vectors standing for eigenmode amplitude of forward and backward waves, 
respectively. Defining $\Psi$ as a pair of the electromagnetic field for $\Phi$ in (11), it is given by

$$
\begin{aligned}
\{\Psi\} & =j[P] \frac{c}{\omega} \frac{\partial \Phi}{\partial z} \\
= & \frac{c}{\omega}\left\{[P][Q]\left\{\phi^{(+)}\right\} e^{-j[Q] z}\right. \\
& \left.-[P][Q]\left\{\phi^{(-)}\right\} e^{j[Q] z}\right\}
\end{aligned}
$$

with

$$
\begin{aligned}
{[P] } & =\left[M_{0}\right]^{-1}[M] \\
{\left[M_{0}\right] } & =\sum_{e} \int\{N\}\{N\}^{T} d y .
\end{aligned}
$$

The boundary condition of a certain electromagnetic field at the discontinuity boundary can be expressed as follows in a matrix form:

$$
\left[\begin{array}{cc}
{[I]} & {[I]} \\
{\left[Z_{L}\right]} & -\left[Z_{L}\right]
\end{array}\right]\left[\begin{array}{l}
\left\{\phi_{L}^{(+)}\right\} \\
\left\{\phi_{L}^{(-)}\right\}
\end{array}\right]=\left[\begin{array}{cc}
{[I]} & {[I]} \\
{\left[Z_{R}\right]} & -\left[Z_{R}\right]
\end{array}\right]\left[\begin{array}{l}
\left\{\phi_{R}^{(+)}\right\} \\
\left\{\phi_{R}^{(-)}\right\}
\end{array}\right]
$$

where $\left[Z_{i}\right](i=L, R)$ is defined by $\left[P_{i}\right]\left[Q_{i}\right]$ and the subscripts $L$ and $R$ indicate the input waveguide side and the output waveguide side, respectively. From (15), if there is no backward wave in the output side waveguide, namely $\left\{\phi_{R}^{(-)}\right\}=\{0\}$, we can derive the reflection amplitude as follows:

$$
\begin{aligned}
& \left\{\phi_{L}^{(-)}\right\}=\frac{\left[Z_{L}\right]-\left[Z_{R}\right]}{\left[Z_{L}\right]+\left[Z_{R}\right]}\left\{\phi_{L}^{(+)}\right\} \\
& \left\{\phi_{R}^{(+)}\right\}=\left\{\phi_{L}^{(+)}\right\}+\left\{\phi_{L}^{(-)}\right\}
\end{aligned}
$$

where $\left[Z_{i}\right](i=L, R)$ can be regarded as an impedance operator or an admittance operator depending on whether $\phi$ is an electric field or magnetic field.

\section{BPM Analysis}

Substituting a solution of the form

$$
\Phi(y, z)=\phi(y, z) \exp \left(-j k_{0} n_{0}\right)
$$

into (1), we obtain the following equation for the slowly varying complex amplitude $\phi$ :

$\frac{\partial}{\partial y}\left(\frac{p}{s_{y}} \frac{\partial \phi}{\partial y}\right)+s_{y} \frac{\partial^{2} \phi}{\partial z^{2}}-j 2 k_{0} n_{0} s_{y} p \frac{\partial \phi}{\partial z}+k_{0}^{2}\left(s_{y} q-n_{0}^{2} p\right) \phi=0$

where $n_{0}$ is the reference refractive index. Then, dividing the waveguide cross-section into the line elements, applying the FEM to (18), and using Padé approximation, we obtain

$$
\begin{aligned}
& -j 2 k_{0} n_{0}[\tilde{M}] \frac{d\{\phi\}}{d z} \\
& \quad+\left([K]-k_{0}^{2} n_{0}^{2}[M]\right)\{\phi\}=\{0\} \\
& {[\tilde{M}]=[M]+\frac{1}{4 k_{0}^{2} n_{0}^{2}}\left([K]-k_{0}^{2} n_{0}^{2}[M]\right) .}
\end{aligned}
$$

Applying the $\theta$-scheme for the propagation direction $z$ to (19) yields

$$
[A]_{i}\{\phi\}_{i+1}=[B]_{i}\{\phi\}_{i}
$$

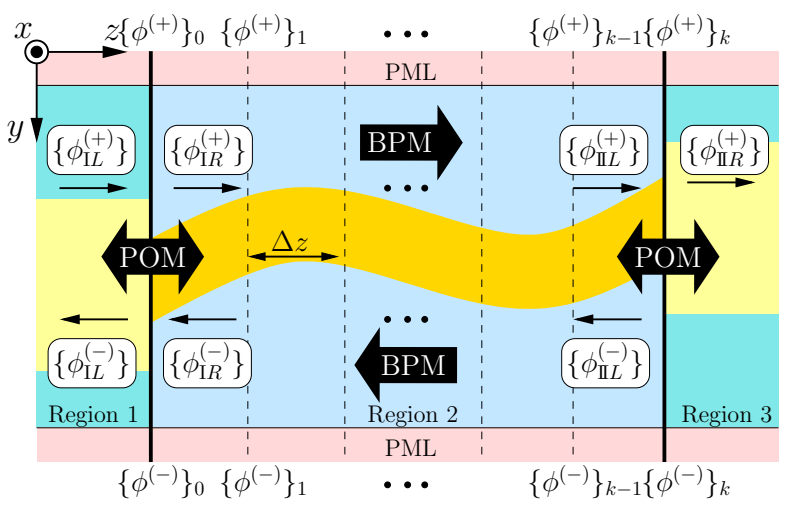

Fig. 1. Combination of POM and BPM.

with

$$
\begin{aligned}
& {[A]_{i}=-j 2 k_{0} n_{0}[\tilde{M}]_{i}+\theta \Delta z\left([K]_{i}-k_{0}^{2} n_{0}^{2}[M]_{i}\right)} \\
& {[B]_{i}=-j 2 k_{0} n_{0}[\tilde{M}]_{i}+(\theta-1) \Delta z\left([K]_{i}-k_{0}^{2} n_{0}^{2}[M]_{i}\right) .}
\end{aligned}
$$

From (22), when the electromagnetic field at the incident position $\{\phi\}_{0}$ is given, the field amplitude at an arbitrary position $z=i \Delta z$ is obtained by sequential calculation.

\section{Combining POM and BPM}

Now, we consider a structure where two discontinuous cross-sections are included in the waveguide as shown in Fig. 1. Dividing the region where it is assumed that the structure gradually varies in the propagation direction into $k$ subregions, the relationship between the forward wave and the backward wave at both ends of the region can be expressed by

$$
\begin{aligned}
& \left\{\phi_{\mathrm{IR}}^{(+)}\right\}=\prod_{i}[B]_{i}^{-1}[A]_{i}\left\{\phi_{\Pi L}^{(+)}\right\} \\
& \left\{\phi_{\mathrm{I} R}^{(-)}\right\}=\prod_{i}[A]_{i}^{-1}[B]_{i}\left\{\phi_{\Pi L}^{(-)}\right\}
\end{aligned}
$$

and combining these equations given by BPM and relationship of POM like (15), we can obtain the final evaluation formula as below

$$
\begin{aligned}
{\left[\begin{array}{l}
\left\{\phi_{\mathrm{IL}}^{(+)}\right\} \\
\left\{\phi_{\mathrm{IL}}^{(-)}\right\}
\end{array}\right] } & =\left[P_{\mathrm{I}}\right][B]\left[P_{\mathrm{II}}\right]\left[\begin{array}{c}
\left\{\phi_{\mathrm{IIR}}^{(+)}\right\} \\
\{0\}
\end{array}\right] \\
{\left[P_{\mathrm{I}}\right] } & =\left[\begin{array}{cc}
{[I]} & {[I]} \\
{\left[Z_{\mathrm{I} L}\right]} & -\left[Z_{\mathrm{I} L}\right]
\end{array}\right]^{-1}\left[\begin{array}{cc}
{[I]} & {[I]} \\
{\left[Z_{\mathrm{I} R}\right]} & -\left[Z_{\mathrm{I} R}\right]
\end{array}\right] \\
{[B] } & =\left[\begin{array}{cc}
\prod_{i}[B]_{i}^{-1}[A]_{i} & {[0]} \\
{[0]} & \prod_{i}[A]_{i}^{-1}[B]_{i}
\end{array}\right] \\
{\left[P_{\mathrm{II}}\right] } & =\left[\begin{array}{cc}
{[I]} & {[I]} \\
{\left[Z_{\mathbb{I I L}}\right]} & -\left[Z_{\mathbb{I I} L}\right]
\end{array}\right]^{-1}\left[\begin{array}{cc}
{[I]} & {[I]} \\
{\left[Z_{\Pi L R}\right.} & -\left[Z_{\mathbb{I I} R}\right]
\end{array}\right]
\end{aligned}
$$

where the subscripts I and II represent each boundaries with different structures. If the waveguide structures of the input side and the output side are the same, $\left[P_{\mathrm{I}}\right]$ is the inverse matrix of $\left[P_{\mathrm{I}}\right]$. Besides, when there are three or more discontinuous cross-section, the transfer matrices obtained by BPM and POM can be connected in multiple stages in the same way. 


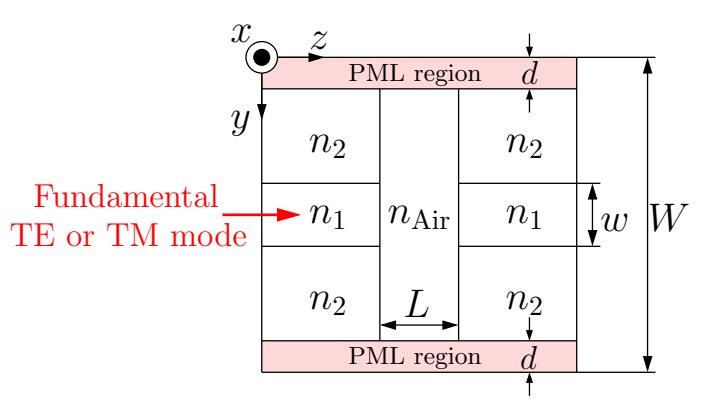

Fig. 2. Problem setup of 2-D optical waveguide with an air-gap.

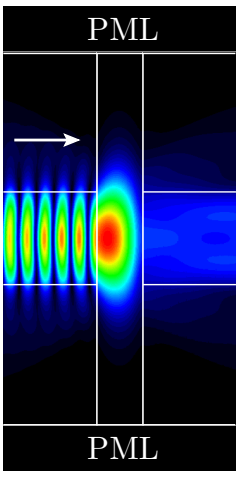

(a)

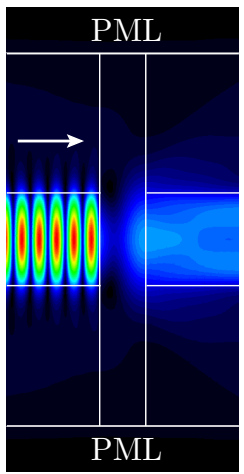

(b)
Fig. 3. Propagation field distribution obtained by FEM analysis when $n_{1}=$ 3.54, $n_{2}=3.17$ and $L=0.5 \mu \mathrm{m}$. Incident field: (a) fundamental TE mode and (b) fundamental TM mode.

\section{NUMERICAL SIMULATION RESULTS}

\section{A. Weakly guiding waveguides with an air-gap}

In this section, in order to confirm the validity of the proposed method described above, waveguides having two discontinuous cross-sections as shown in Fig. 2 is analyzed by the proposed method. We consider the problem that an air-gap caused between the waveguides, and input and output waveguides are assumed to have a same structure. First, weakly guiding waveguides with core index $n_{1}=3.54$, cladding index $n_{2}=3.17$, core width $w=1 \mu \mathrm{m}$ is considered. The computational window size is $W=5 \mu \mathrm{m}$ and spurious reflection from the end of the analysis region is suppressed by imposing a perfectly matched layer (PML) [30] on the width $d=0.5 \mu \mathrm{m}$ from the end of the analysis region.

Figure 3 shows the propagation field distribution calculated by FEM when the air-gap length $L=0.5 \mu \mathrm{m}$. In Fig. 3, since the electric field is drawn for the TE mode and the magnetic field for the TM mode, the amplitude of the field propagating in the air-gap is smaller in the TM mode than in the TE mode. Since there is no structure to confine light at the gap region, it is seen that the light radiated to the air-gap is widely spreading in the lateral direction and various modes are excited. In this case, a simple evaluation scheme by overlap integral which widely used in the analysis of the butt-coupling between weakly guiding waveguides is insufficient and a more rigorous analysis method is required.

Figure 4 shows the reflection of the fundamental mode

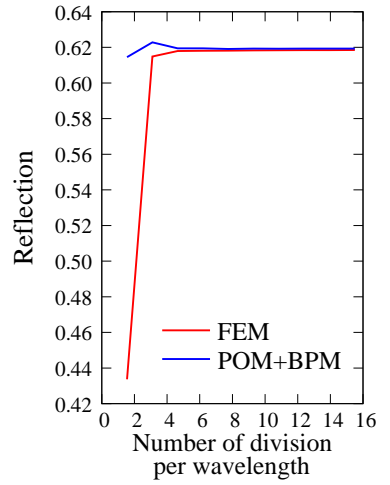

(a)

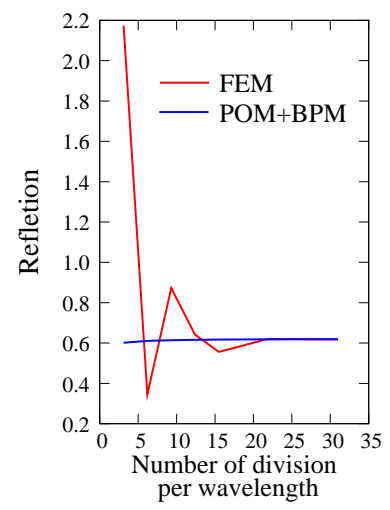

(b)
Fig. 4. Reflection of the fundamental mode as a function of number of discretization per wavelength; (a) convergence behavior with respect to discretization number along transverse direction: (b) convergence behavior with respect to discretization number along longitudinal dirction.

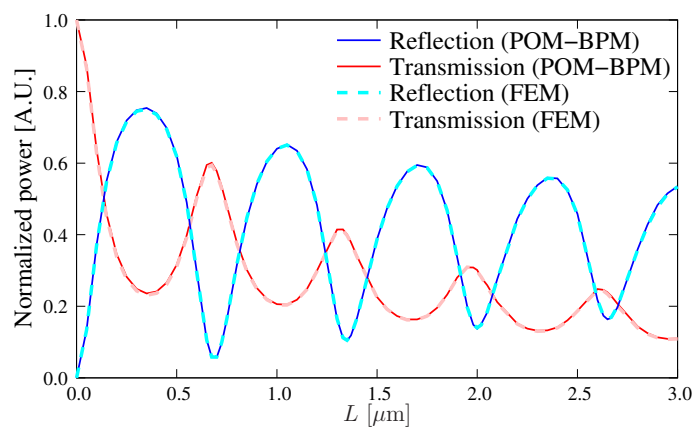

(a)

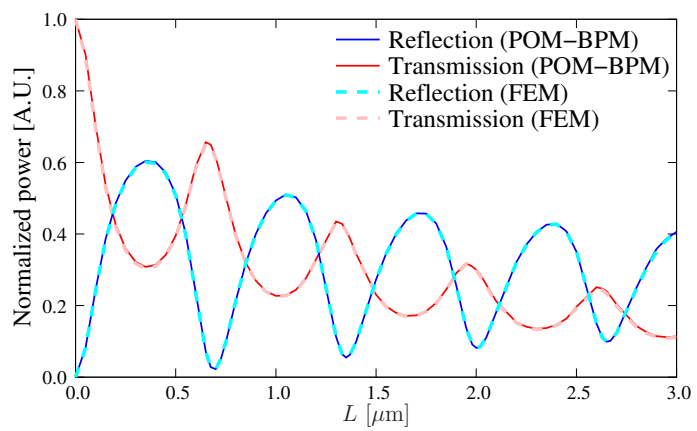

(b)

Fig. 5. Normalized reflected and transmitted power of the fundamental modes as a function of the air-gap length when $n_{1}=3.54, n_{2}=3.17$. Incident field: (a) fundamental TE mode and (b) fundamental TM mode.

as a function of number of discretization per wavelength. Since FEM requires discretization of the entire analysis region, the length of analysis region along the longitudinal direction $L$ is set to $2.5 \mu \mathrm{m}$ including the $0.5 \mu \mathrm{m}$ of PML region at the both ends. We employ uniform mesh with 2nd-order triangular elements in FEM, and also 2nd-order line elements are employed in the presented method. Figure 4(a) shows the convergence behavior as a function of the discretization number along transverse direction when the discretization along the longitudinal direction is sufficiently fine. We can see that the required discretization along the transverse direction is almost same in both FEM and the present method. The 


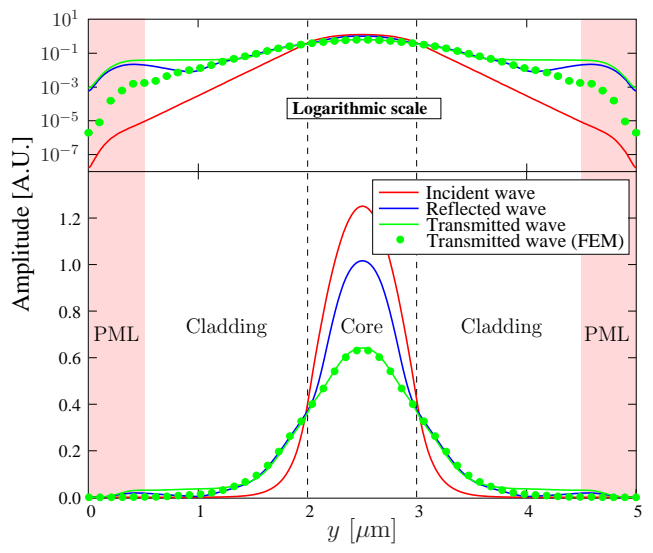

(a)

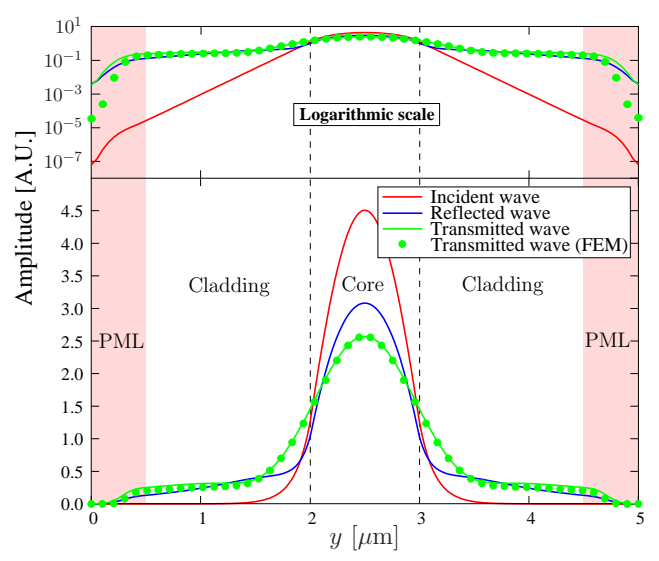

(b)

Fig. 6. Electromagnetic field distribution at discontinuity boundary when $n_{1}=3.54, n_{2}=3.17$ and $L=0.5 \mu \mathrm{m}$. Incident field: (a) fundamental TE mode and (b) fundamental TM mode.

good convergence is obtained with about 10 elements per wavelength. On the other hand, in the present method requires less longitudinal discretization than FEM when the transverse direction is sufficiently discretized, as shown in Fig. 4(b). In this example, the computational times (and the size of matrix to be solved) are $25 \mathrm{~ms}$ (130 unknowns) and $60 \mathrm{~ms}$ (5265 unknowns) in the present method and FEM, respectively. Of cause, the matrix constructed in the FEM is coarse and may be efficiently solved and the computational times are not much different with each other in this example. However, in the case of three-dimensional vector wave analysis, the present method is expected to be a more efficient method than FEM due to the large reduction of discretization [17]. Moreover, since it is possible to calculate the transfer matrix in parallel at the structure of each waveguide in POM, further efficiency improvement can be expected.

Figure 5 shows the normalized reflected power and transmitted power of the fundamental modes as a function of the air-gap length $L$ when the fundamental TE or TM mode with operating wavelength of $1.3 \mu \mathrm{m}$ is launched. From this figure, it can be seen that the transmission and the reflection become stronger or weaker while the air-gap length varies. This is a phenomenon caused by Fabry-Perot resonance between two discontinuous boundaries, and it can be confirmed that

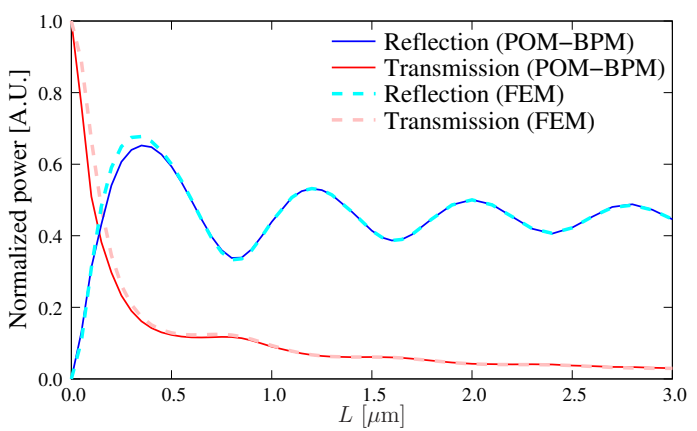

(a)

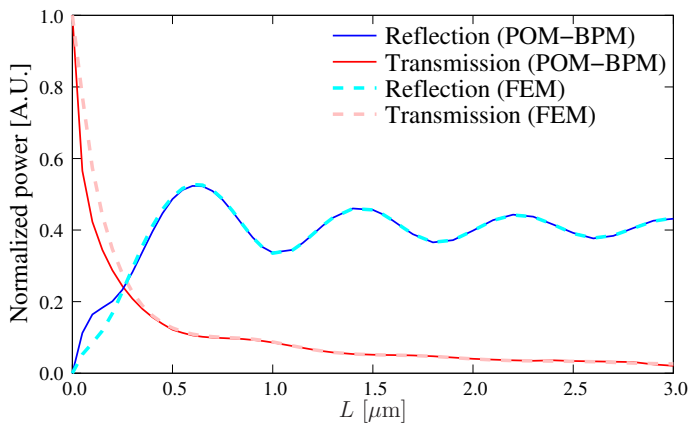

(b)

Fig. 7. Normalized reflected and transmitted power of the fundamental modes as a function of the gap length when $n_{1}=3.6, n_{2}=1$. Incident field: (a)fundamental TE mode and (b) fundamental TM mode.

the proposed method considers the interference of the light reflected and transmitted at the discontinuous boundaries. This figure also shows the results obtained by FEM for comparison, and it shows that it agrees well with the result of our method.

The electromagnetic field distribution for $L=0.5 \mu \mathrm{m}$ is shown in Fig. 6. In terms of reflected waves and transmitted waves, light spreads to the cladding region and it is seen that the radiation field can be evaluated by the proposed method. In the field distribution with respect to the incident field of fundamental TM mode, the light spreads to the far side in the transverse direction compared to the results of TE mode, and simultaneously it is absorbed by PML imposed on the end of the analysis region for suppressing the spurious reflection. For the transmitted waves, the results of FEM with more strict accuracy by discretizing the entire analysis region is also depicted in Fig. 6, and it agree well with the result of the proposed method in the core region. Although a slight error is caused in the cladding region between FEM and our method, as shown in Fig. 5, the error hardly affected for evaluating the propagation characteristics of the fundamental mode, and it is said that the analysis accuracy of the proposed method can sufficiently offer the actual requirement of the evaluation criteria.

\section{B. Strongly guiding waveguides with an air-gap}

Next, strongly guiding waveguides with core index $n_{1}=$ 3.6 , cladding index $n_{2}=1$, core width $w=0.5 \mu \mathrm{m}$ is considered. The computational window size is $W=3.0 \mu \mathrm{m}$ and PML is imposed at the end of analysis region with the 


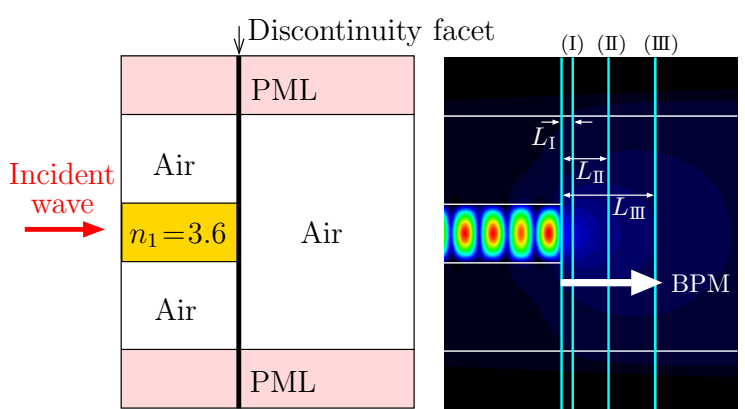

(a)
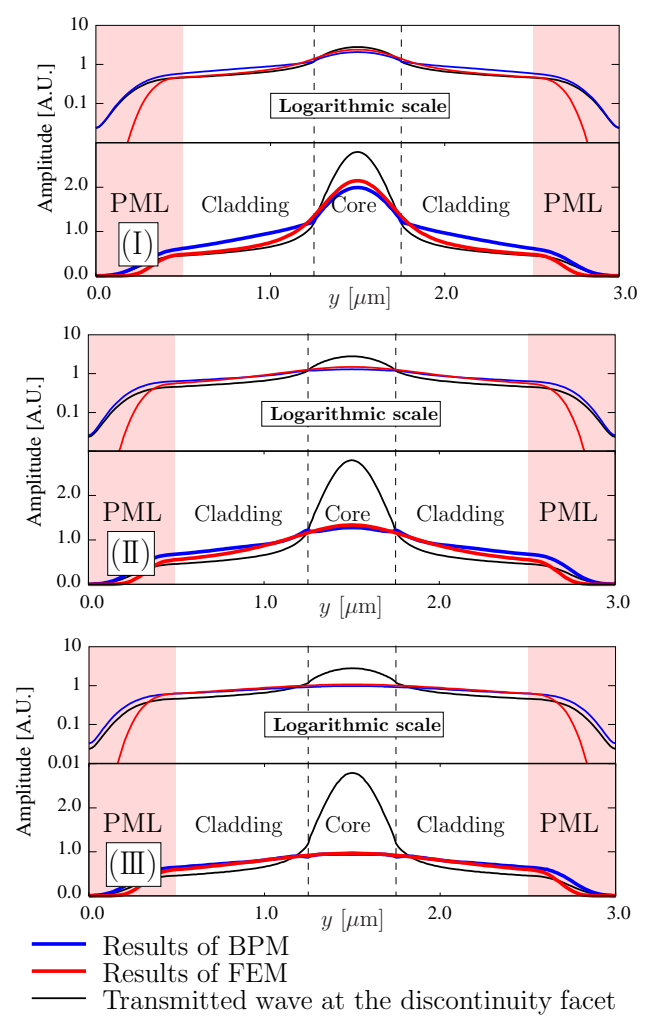

(b)

Fig. 8. Optical radiation from end facet of 2-D waveguide when fundamental TM mode is launched: (a) propagation field distribution of magnetic field and (b) magnetic field amplitude at propagation length $L_{I}=0.1 \mu \mathrm{m}, L_{I I}=$ $0.4 \mu \mathrm{m}$ and $L_{I I I}=0.8 \mu \mathrm{m}$ calculated by BPM and FEM.

same width as the above problem. Here, since the cladding material is assumed to be the same as the air-gap, the diffraction of light is likely to occur in the cladding region when the light is radiated to the air from the input waveguide, and thus, analysis with higher accuracy than the weakly guiding waveguide problem described above is required.

Figure 7 shows the normalized reflected power and transmitted power of the fundamental modes as a function of the air-gap length $L$ when the fundamental TE or TM mode with operating wavelength of $1.55 \mu \mathrm{m}$ is launched. Although it is found that the reflection and transmission are in good agreement with FEM results when the gap length is relatively long, they do not coincide in both TE and TM mode when the gap length is short.

To study the reason, we investigate the light propagating characteristics of fundamental TM mode radiated from a single discontinuity facet using BPM, and compared with the analysis result of FEM as shown in Fig. 8. This figure shows the electromagnetic field distribution of the waveguide cross-section propagated from discontinuous boundary to the air by the distance $L_{\mathrm{I}}=0.1 \mu \mathrm{m}, L_{\mathrm{II}}=0.4 \mu \mathrm{m}$ and $L_{\mathrm{III}}=0.8 \mu \mathrm{m}$. When the propagation distance is short, the error between BPM and FEM is large, and as the propagation distance becomes longer, the error becomes smaller. Comparing amplitudes obtained by BPM and FEM, evanescent waves which is attenuated with propagation on the cladding region propagates without attenuation in BPM. This problem is considered to be due to the fact that it can not be evaluated correctly for the wavevector which are greatly different from the propagation direction in approximation of BPM, as a result, deterioration of accuracy in Fig. 7 is probably caused. On the other hand, at the distance where the evanescent wave is sufficiently attenuated, the result of the proposed method is quite close to the results of FEM, and it can be handled with high accuracy by this method. Since BPM is applied to a uniform medium in the propagation direction here, it is easy to create a transfer matrix using eigenmode expansion instead of using BPM. However, when the structure varies in the propagation direction, the computational cost becomes enormous for the eigenmode expansion. It has been proposed to suppress excitation of unnecessary modes by utilizing a linear filter in full-vectorial finite element BPM [28], and it is considered that undesired modes can be suppressed by applying this filter to our method. However, it is difficult to set the filtering range because it is necessary to handle various radiation mode in the problem discussed here. Nevertheless, since it can be calculated with relatively low calculation cost by FEM when the propagation distance is short, the proposed method is still very useful for analysis of optical waveguides with long propagation distances.

\section{A taperd waveguide between uniform waveguides}

In the above two analysis examples, the BEP can be easily applied because there are only two structures required for eigenmode expansion and we confirm that same results are obtained. Here, a tapered waveguide whose structure varies continuously between two different uniform waveguides as shown in Fig. 9 is analyzed. In order to show the applicability to problems with significant multiple reflection at discontinuities, the uniform waveguide with a core index of 3.24 and cladding index of 3.17 , and the tapered waveguide with a core index of 1.45 and cladding index of 1.445 is employed. Such a problem is difficult tot be evaluated by conventional BPM. The fundamental mode with operating wavelength of $1.55 \mu \mathrm{m}$ is launched into a left side uniform waveguide with a core width of $1 \mu \mathrm{m}$, and then, the light propagates bound for a uniform waveguide with a core width of $4 \mu \mathrm{m}$ via a tapered waveguide whose width is linearly varying from $2 \mu \mathrm{m}$ to $3 \mu \mathrm{m}$.

The electromagnetic field distribution at the input and output ports is shown in Fig. 10. The inset in Fig. 10 shows the electromagnetic field distribution calculated by FEM, and it can be seen that the light is strongly reflected from the discontinuity 


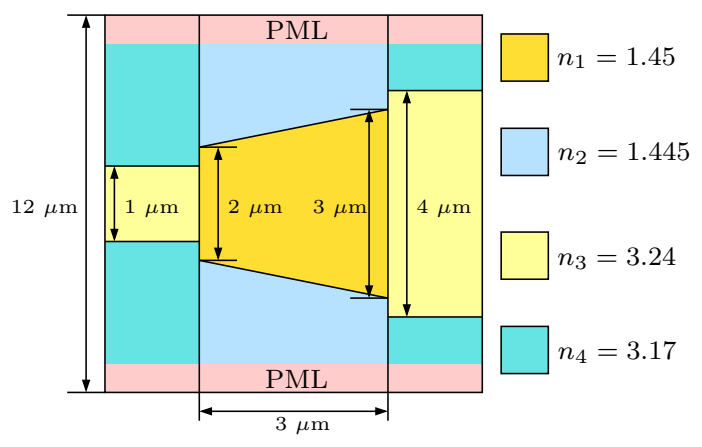

Fig. 9. Taper waveguide between uniform waveguides.

facets and a standing wave is observed. From this figure, we can see that the obtained transmitted waves by the present method and FEM agree well except for the PML region. The transmitted powers obtained by the present method and FEM are $44.23 \%$ and $44.68 \%$ in the case of TE mode and $45.34 \%$ and $45.80 \%$ in the case of TM mode, respectively. It seems that the slight difference of the electromagnetic field in the vicinity of the PML region is due to the fact that the analysis accuracy of BPM for the light radiated in the transverse direction is not sufficient. However, the electromagnetic field is sufficiently attenuated near the end of analysis region. There is no major influence to the evaluation of transmission power. On the other hand, although the analysis result by using conventional BPM which cannot handle the reflection is also shown in the same figure, it is greatly different from the result of this method or FEM. The transmitted power obtained by BPM is $77.78 \%$ and $77.74 \%$ for TE mode and TM mode, respectively, which are optimistically evaluated.

\section{CONCLUSION}

We proposed a combination method of POM and BPM based on FEM for multiple waveguide discontinuity problem and investigated its validity. In the numerical example assuming the case where the air-gap occurs between the optical waveguides, it was shown that high precision analysis can be performed except for analysis of a structure with a short gap length in a strongly guiding waveguide problem. This remaining matter was shown to be due to the inaccuracy for evaluation of the evanescent wave offered by BPM approximation. The proposed method is very useful for discontinuity problems with a long propagation distance and high refractive index difference, and the efficiency will be dramatically increased when it is applied to the analysis of three-dimensional waveguide which requires a huge amount of computational cost, and this is our future work.

\section{REFERENCES}

[1] Y. Tsuji, K. Hirayama, T. Nomura, K. Sato, and S. Nishiwaki, "Design of optical circuit devices based on topology optimization," IEEE Photon. Technol. Lett., vol. 18, no. 7, pp. 850-852, April 2006.

[2] L. H. Frandsen, Y. Elesin, L. F. Frellsen, M. Mitrovic, Y. Ding, O. Sigmund, and K. Yvind, "Topology optimized mode conversion in a photonic crystal waveguide fabricated in silicon-on-insulator material," Opt. Express, vol. 22, no. 7, pp. 8525-8532, April 2014.

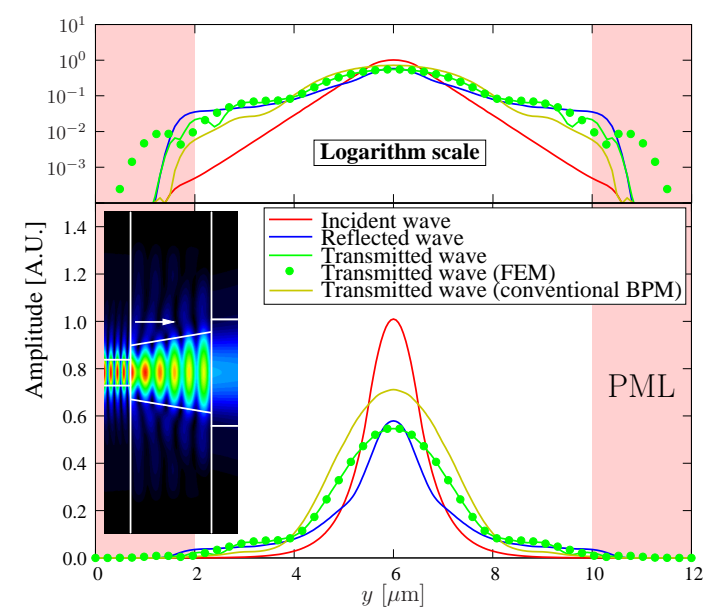

(a)

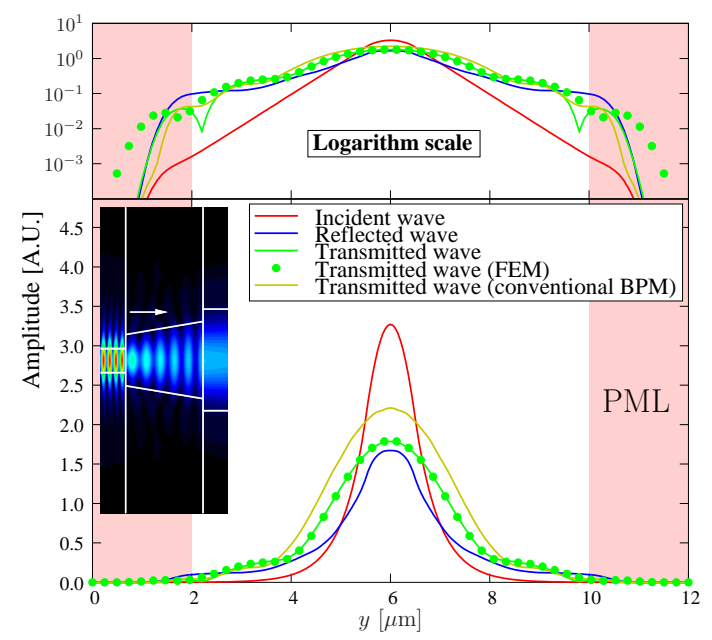

(b)

Fig. 10. Electromagnetic field distribution at the input and output ports corresponding to the problem shown in Fig. 9. The inset is a propagating field obtained by FEM; Incident field: (a) fundamental TE mode and (b) fundamental TM mode.

[3] Z. Zhang, Y. Tsuji, T. Yasui, and K. Hirayama, "Design of ultra-compact triplexer with function-expansion based topology optimization," Opt. Express, vol. 23, no. 4, pp. 3936-3950, Feb. 2015.

[4] A. Iguchi, Y. Tsuji, and K. Hirayama, "Topology optimization of optical waveguide devices based on beam propagation method with sensitivity analysis," J. Lightw. Technol., vol. 34, no. 18, pp. 4214-4220, Sept. 2016.

[5] A. Iguchi, Y. Tsuji, T. Yasui, and K. Hirayama, "Efficient topology optimization of optical waveguide devices utilizing semi-vectorial finitedifference beam propagation method," Opt. Express, vol. 25, no. 3, pp. 28210-28222, Nov. 2017.

[6] M. Koshiba, Optical Waveguide Theory by the Finite Element Method. Tokyo, Japan/Dordrecht, The Nethierland: KTK Scientific/Kluwer Academic, 1992.

[7] Y. Tsuji and M. Koshiba, "Finite element method using port truncation by perfectly matched layer boundary conditions for optical waveguide discontinuity problems," J. Lightw. Technol., vol. 20, no. 3, pp. 463-468, March 2002.

[8] K.S. Yee, "Numerical solution of initial boundary value problems involving Maxwell' s equations," IEEE Trans. Antennas Propag., vol. AP-14, pp. 302-307, May 1966.

[9] G. Sztefka and H. P. Nolting, "Bidirectional eigenmode propagation for large refractive index steps," IEEE Photon. Technol. Lett., vol. 5, no. 5, pp. 554-557, May 1993.

[10] P. Bienstman and R. Baets, "Optical modelling of photonic crystals and VCSELs using eigenmode expansion and perfectly matched layers," Opt. Quantum Electron., vol. 33. no. 4-5, pp. 327-341, April 2001. 
[11] , S. Helfert, R. Pregla, P. Bienstman, R. Baets, R. De Ridder, R. Stoffer, G. Klaasse, J. Petrek, and P. Lalanne, "Bragg waveguide grating as a $1 \mathrm{~d}$ photonic band gap structure: COST 268 modelling task," Opt. Quantum Electron., vol. 34, no. 5-6, pp. 455-470, May 2002.

[12] H. El-Refaei, I. Betty, and D. Yevick, "The application of complex Padé approximants to reflection at optical waveguide facets," IEEE Photon. Technol. Lett., vol. 12, no. 2, pp. 158-160, Feb. 2000.

[13] H. Zhang, Q. Guo, and W. P. Huang, "Analysis of waveguide discontinuities by a fourth-order finite-difference reflective scheme," J. Lightw. Technol., vol. 25, no. 2, pp. 556-561, Feb. 2007.

[14] H. Jamid and M. Khan, "3-D full-vectorial analysis of strong otical waveguide discontinuities using Padé approximants," IEEE J. Quantum Electron., vol. 43, no. 4, pp. 343-349, Apr. 2007.

[15] S. Wu, J. Xiao, and X. Sun, "Full-vectorial analysis of optical waveguide discontinuities using Denman-Beavers iterative scheme," J. Lightw. Technol., vol. 33, no. 2, pp. 511-518, Jan. 2015.

[16] K. Morimoto and Y. Tsuji, "Analysis of butt coupling of optical waveguides using propagation operator method based on finite element method," IEICE Trans. Electron. (Japanese Edition), vol. J101-C, no. 5, pp. 210-216, May 2018.

[17] K. Morimoto and Y. Tsuji, "Full-vectorial analysis of optical waveguide discontinuities using propagation operator method based on finite element method," OSA Continuum, vol. 2, no. 3, pp. 540-553, March 2019.

[18] M. Scalora and M. E. Crenshaw, "A beam propagation method that handles reflections," Opt. Commun., vol. 108, no. 4-6, pp. 191-196, June 1994.

[19] Y. Tsuji and M. Koshiba, "Noniterative beam propagation method for optical grating waveguides," Electron. Commun. Jap. Part II- Electron., vol. 81, no. 8, pp. 49-54, Aug. 1998.

[20] Y. Y. Lu and S. H. Wei, "A new iterative bidirectional beam propagation method," IEEE Photon. Technol. Lett., vol. 14, no. 11, Nov. 2002.

[21] H. El-Refaei, D. Yevick, and I. Betty, "Stable and noniterative bidirectional beam propgation method," IEEE Photon. Technol. Lett., vol. 12, no. 4, pp. 389-391, April 2000.

[22] H. Zhang, J. Mu, and W.-P. Huang, "Improved bidirectional beampropagation method by a fourth-order finite-difference scheme," $J$. Lightw. Technol., vol. 25, no. 9, pp. 2807-2813, Sept. 2007.

[23] J. Xiao, S. Wu, and X. Sun, "A Stable and Accurate Preconditioner for bidirectional beam propgation method," IEEE J. Quantum Electron., vol. 51, no. 4, April 2015.

[24] S. S. A. Obayya, Computational Photonics (John Wiley \& Sons, 2011).

[25] W. P. Huang, C. L. Xu, S. T. Chu, and S. K. Chaudhuri,"A vector beam propagation method for guided-wave optics," IEEE Photon. Technol. Lett., vol. 3, no. 10, pp. 910-913, Oct. 1991.

[26] Y. Tsuji, M. Koshiba, and T. Shiraishi, "Finite element beam propagation method for three-dimensional optical waveguide structures," J. Lightw. Technol., vol. 15, no. 9, pp. 1728-1734, Sep. 1997.

[27] E. Montanari, S. Selleri, L. Vincetti, and M. Zoboli, "Finite-element fullvectorial propagation analysis for three-dimensional z-varying optical waveguides," J. Lightw. Technol., vol. 16, no. 4, pp. 703-714, April 1998.

[28] S. Kawai, A. Iguchi, and Y. Tsuji, "Study on high precision and stable finite element beam propagation method based on incomplete third order hybrid edge/nodal element," J. Lightw. Technol., vol. 36, no. 11, pp. 2278-2285, June 2018.

[29] K. Hayashi, M. Koshiba, Y. Tsuji, S. Yoneta, and R. Kaji, "Combination of beam propagation method and mode expansion propagation method for bidirectional optical beam propagation analysis," J. Lightw. Technol., vol. 16, no. 11, pp. 2040-2045, Nov. 1998.

[30] J.-P. Berenger, "A perfectly matched layer for the absorption of electromagnetic waves," J. Computational Phys., vol. 114, pp. 185-200, Oct. 1994.

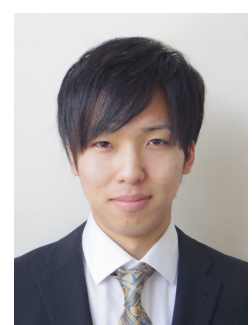

Keita Morimoto received the B.S. and M.S. degree in information and electronic engineering from Muroran Institute of Technology, Muroran, Japan, in 2017 and 2019, respectively, where he is currently worling toward the Ph.D. degree in information and electronic engneering.

Mr. Morimoto is a student member of the Institute of Electronics, Information and Communication Engineers (IEICE).

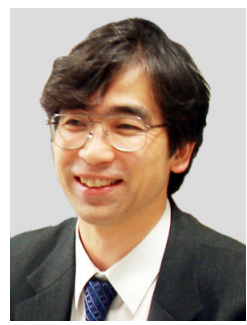

yasuhide Tsuji (M'97) received the B.S., M.S., and $\mathrm{Ph} . \mathrm{D}$. degrees in electronic engineering from Hokkaido University, Sapporo, Japan, in 1991, 1993. and 1996, respectively.

In 1996, he joined the Department of Applied Electronic Engineering, Hokkaido Institute of Technology, Sapporo, Japan. From 1997 to 2004, he was an Associate Professor of Electronics and Information Engineering at Hokkaido University. From 2004 to 2011, he was an Associate Professor of Electrical and Electronic Engineering at Kitami Institute of Technology, Kitami, Japan. Since 2011, he has been a Professor of Information and Electronic Engineering at Muroran Institute of Technology, Muroran, Japan. He has been interested in wave electronics.

Dr. Tsuji is a member of the Institute of Electronics, Information and Communication Engineers (IEICE), the Japan Society of Applied Physics, the Optical Society of America (OSA), and IEEE. In 1997, 1999, and 2019, he was awarded the Excellent Paper Award from IEICE. In 2000, he has received the Third Millennium Medal from IEEE. 